
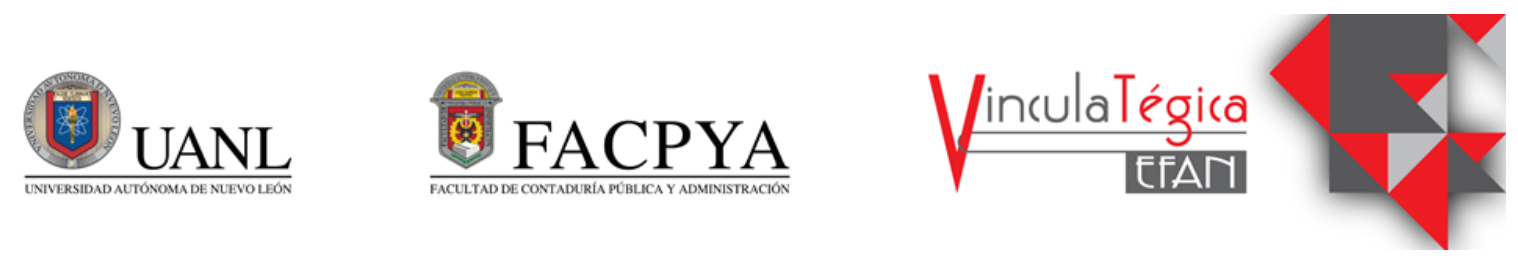

\title{
Participación ciudadana en el ejercicio del derecho de acceso a la información pública en México
}

\author{
María del Carmen Morales Tostado ${ }^{1}$, Reyna Guadalupe Morales Tostado ${ }^{2}$ y Maricela Huerta \\ Salomón $^{3}$ \\ ${ }^{1}$ Universidad de Sonora, carmen.morales@unison.mx, Blvd. Luis Encinas y Rosales. 6621390797. \\ ${ }^{2}$ Universidad de Sonora, reyna.morales@unison.mx, Blvd. Luis Encinas y Rosales 6622571134 \\ ${ }^{3}$ Universidad de Sonora, marisela.huerta@unison.mx.6621831997.
}

Información del artículo revisado por pares

Fecha de aceptación: junio-2021

Fecha de publicación en línea: diciembre-2021

DOI: https://doi.org/10.29105/vtga7.1-105

\section{Resumen}

La presente investigación, abordó el ejercicio del derecho de acceso a la información pública en México y tiene como objetivo brindar información a los interesados sobre el derecho de acceso a la información pública, su estructura jurídica y la participación de los ciudadanos en el ejercicio de ese derecho. Por el tema a investigar, se eligió el enfoque cualitativo y el método descriptivo, para lograr los objetivos y se investigó las solicitudes que se recibieron en el Instituto Nacional de Transparencia y Acceso a la Información Pública y los sujetos obligados que recibieron mayor número de solicitudes Después de analizar la temática que nos ocupa, se llegaron a los siguientes resultados, las evoluciones de las leyes brindan certidumbre jurídica a los usuarios de derecho de acceso a la información. El gobierno mexicano enfrenta muchos retos, quizá de mayor prioridad que el brindar acceso a la información pública, como el aumento de los ciudadanos que viven en pobreza, la inseguridad, la contingencia sanitaria por COVID 19, los procesos electorales costosos, entre otros. Dentro de las conclusiones, se identifica que se han logrado avances significativos en el marco jurídico, sin embargo, hay mucho por hacer para que se observen cambios en la cultura de los ciudadanos. La creación y modificaciones de las leyes en la materia, han coadyuvado a que se presente un incremento de la participación ciudadana, tal como se refleja en las solicitudes de información que recibe el Instituto Nacional de Transparencia.

\section{Abstract}

This investigation addressed the exercise of the right of access to public information in México and aims to provide information to interested parties on the right of access to public information, its legal structure and the participation of citizens in the exercise of that 
right. For the topic to be investigated, the qualitative approach and the descriptive method were chosen to achieve the objectives and the requests received at the National Institute for Transparency and access to public information and the obligated subjects who received the highest number of requests were investigated. After analyzing the issue at hand, the following results were reached: the evolution of the laws provide legal certainty to users of the right of access to information. The Mexican government faces many challenges, perhaps of higher priority tan providing access to public information, such as the increase in citizens living in poverty, insecurity, the health contingency due to COVID 19, costly electoral process, among others. Within the conclusions, it is identified that significant progress has been made in the legal framework; however, there is much to do to see changes in the culture of citizens. The creation and modifications of the laws on the subject have assisted to an increase in citizen's participation, as reflected in the requests for information receives by the National Institute of Transparency.

Palabras clave: Participación ciudadana, derecho acceso a la información pública y gobierno transparente.

Key words: Citizen participation, right of Access to public information and transparent government.

\section{Código JEL}

H: Economía pública. H11: Estructura, ámbito y funcionamiento del sector público

\section{INTRODUCCIÓN}

La temática sobre transparencia y acceso a la información pública, resulta interesante ya que los ciudadanos no tiene confianza en el gobierno, por los repetidos actos de corrupción que se dan a conocer en los medios de comunicación.

El derecho de acceso a la información gubernamental se considera como uno de los derechos humanos plasmado en el contexto internacional en Francia en la Declaración de los Derechos del Hombre y Ciudadano desde 1789. En la República Mexicana se consagró en la Constitución Política de los Estados Unidos Mexicanos en 1917. Sin embargo, la primera Ley de Transparencia se aprobó en 2002 y una segunda Ley de Transparencia con mayor certidumbre jurídica se promulgó en 2015.

En estos procesos de transformación del gobierno, que transita de un gobierno cerrado al gobiero abierto y que rinda cuentas, ha sido determinante la participación de la sociedad civil. En el contexto social y político permea la falta de confianza en las instituciones públicas, por tanto, la transparencia se presenta como estrategia para construir la confianza de los ciudadanos y puede contribuir a un mejor desempeño de los servidores públicos.

Para avanzar se requiere, una participación ciudadana activa, tanto en el monitoreo, como en el seguimiento y la evaluación del desempeño de las instituciones públicas, así como, en el establecimiento de medidas correctivas dentro de la función pública.

Dentro de los resultados se presenta que en el Instituto Nacional de Transparencia, Derecho de Acceso a la Información y Protección de Datos Personales (INAI) se han recibido solicitudes de 
información de los ciudadanos interesados en conocer el quehacer gubernamental, donde se observa un incremento cada año.

También, es importante señalar que la creación y modificaciones a las leyes relacionadas, proporcionan mayor certidumbre jurídica para ejercer el derecho de acceso a la información pública.

\section{MARCO TEÓRICO}

Para desarrollar la investigación se realizó una recopilación de información sobre antecedentes y evolución del derecho de acceso a la información, se revisaron investigaciones previas $\mathrm{y}$ las consideraciones teóricas que dan sustento a la investigación.

\subsection{Derecho de acceso a la información pública}

La participación de la sociedad civil en los últimos años, ha exigido un gobierno abierto y transparente, que respete el derecho de los ciudadanos el acceso a la información pública, ya que permite conocer el desempeño del gobierno y de las organizaciones que reciben recursos públicos.

En ese sentido, la Ley de Transparencia, intenta permear en la cultura de los ciudadanos, desde dos contextos totalmente diferentes: el primero es relacionado a la cultura de los servidores públicos para que realmente se reflejen en su conducta los valores que como seres humanos y como servidor público se requieren practicar. Ello, les permitirá proporcionar la información que están obligados a ofrecer a quien lo solicite sin restricción ni pretextos y el segundo es desde el contexto del ciudadano que debe empoderarse y hacer valer el derecho a saber sobre que hace el gobierno.

Por eso, es de interés, que los ciudadanos identifiquen y conozcan el derecho de acceso a la información y que les permite solicitar información en poder del gobierno.

Otro factor primordial es la utilización de las tecnologías de información y comunicación, ya que todo se realiza a través de plataformas digítales y más a partir de marzo de 2020 que inicio la contingencia sanitaria por el COVID 19 en el mundo, es ese tenor es necesario compartir lo planteado por Quintanilla y Gil (2016:72) que dicen:

En la última década, las tecnologías de la información han sido, en mayor o menor medida, instrumentos de reforma administrativa que pueden ayudar a los gobiernos a generar mecanismos para ser más eficientes, transparentes $\mathrm{y}$ participativos.

En este tema, se considera importante señalar lo que comparte la Instituto Nacional de Estadística y Geografía (INEGI), en su comunicado 216/20 de fecha 14 de mayo de 2020, donde según los datos de la Encuesta Nacional sobre Disponibilidad y Uso de Tecnologías de la Información en los Hogares de 2019 (ENDUTIH), el 70.1 por ciento de la población de seis años o más, es usuaria de internet. También, se da a conocer que el 56.4 por ciento de los hogares del país disponen de conexión a internet. Considerando los datos presentados anteriormente, todavía no disponen de internet el $43.6 \%$ de los hogares en la República Mexicana. 


\subsection{Estructura jurídica del derecho de acceso a la información pública.}

El sustento jurídico del derecho de acceso a la información pública, se encuentra en la Constitución Política de los Estados Unidos Mexicanos (CPEUM), donde se incorporó desde 1917. Sin embargo, fue algo olvidado y no se consideró una prioridad por los gobiernos.

Fue hasta el año 2002 cuando se aprobó la ley de transparencia y en ella se establece la obligación de informar a la sociedad y con ello, se da inicio a una nueva relación entre el gobierno y ciudadanos. Así mismo, en mayo de 2015 se publica en el Diario Oficial de la Federación la nueva Ley General de Transparencia y Acceso a la Información Pública (LGTAIP).

El estudio de los antecedentes del derecho de acceso a la información, nos permite conocer que López (2009) señala el documento de mayor antigüedad que data desde 1789 y es parte de la Declaración de los Derechos del Hombre y Ciudadano signado en Francia.

En el contexto internacional, el derecho a la información pública ha permeado en la cultura social y política, convirtiéndose en una corriente universal, y en especial los Estados constitucionales, donde el principio de legalidad constituye la base para su desempeño y para la formulación de políticas donde privilegian el bien común de sus ciudadanos, se ha establecido como resultado una mejor relación entre gobierno y ciudadanos.

Por ello, en México atendiendo el entorno internacional, se han creado y realizado modificaciones a las leyes mexicanas que promueven la participación de los ciudadanos, para ejercer su derecho de acceso a la información que genera el gobierno en su desempeño y también, conocer los motivos en los que se basan para tomar decisiones.

El fundamento legal en la República Mexicana, para hacer uso del derecho de acceso a la información pública, se establecen en la CPEUM en su artículo 6, el cual que ha sido necesario realizar modificaciones para describir en detalle aspectos que quedaban en el limbo jurídico y ahora brinda mayor certidumbre a los ciudadanos que solicita información en poder del gobierno.

Es importante señalar que el ejercicio del derecho de acceso a la información, forma parte de un compromiso internacional para enfrentar las conductas corruptas y en diciembre de 2003 se detalla la estrategia en la Convención de la ONU contra la Corrupción.

\subsection{Participación de la sociedad civil}

La participación de la sociedad civil, se puede analizar desde diferentes perspectivas y en ellas existen varias definiciones de sociedad civil (S.C.), para nuestro propósito se considera a la S.C. como la integración de un grupo de personas que de manera voluntaria se reúnen sin animo de lucro y tienen por objetivo satisfacer necesidades de la sociedad que el gobierno tiene sin cubrir.

El su página electrónica el Instituto Nacional de Desarrollo Social, describe en el menú: Acciones y Programas el programa de Registro Federal de las Organizaciones de la Sociedad Civil (OSC), así como, el siguiente concepto de OSC.

Las organizaciones de la sociedad civil son agrupaciones constituidas por individuos, 
fundamentados en lazos asociativos que pueden realizar actividades de defensa y respeto a los derechos humanos, de apoyo o asistencia a terceros sin fines de lucro ni de proselitismo partidista, político-electoral o religioso, que no persiguen beneficios personales sino sociales comunitarios. Esencialmente su origen responde al derecho de todo miembro de la sociedad de ejercer su participación ciudadana como la clave para la existencia de una mayor corresponsabilidad de los gobiernos y los ciudadanos.

De igual manera, es importante señalar que en la Ley Federal de Fomento a las Actividades de las Organizaciones Civiles promulgada el 9 de febrero de 2004, con modificaciones importantes en 2011, 2012 y las últimas de fecha 24 de abril de 2018. En dicha Ley, se establece en el Artículo 15, la creación del Registro Federal de Organizaciones de la Sociedad Civil.

El Registro tiene por objetivo inscribir a las organizaciones que así lo soliciten y que cumplan con los requisitos que establece la Ley Federal de Fomento a las Actividades Realizadas por Organizaciones de la Sociedad Civil y su reglamento.

Para el dar cumplimiento a la Ley en comento, en lo relacionado al procedimiento para el Registro Federal de las OSC, se crea en el Instituto Nacional de Desarrollo Social, el Portal de las Acciones de Fomento que realiza la Administración Pública Federal para las Organizaciones de la Sociedad Civil, donde se describe en detalle y está disponible en www.corresponsabilidad.gob.mx.

En México, los cambios que se han realizado a las leyes de transparencia ha sido propiciados por la lucha de las S.C., desde la elaboración de la iniciativa de la LFTAIPG en 2002, la sustitución por la LGTAIP en 2015 hasta las modificaciones del artículo 6 constitucional.

Al respecto Luna (2009:163), señala que "la sociedad en México ha respondido de manera lenta asimilando y comprendiendo los usos, alcances y límites del derecho de acceso a la información", quizá esto cambiará en los próximos años, y poco a poco se ira reflejando en la gestión pública. Lo que generará una cultura en los servidores donde se sientan comprometidos con la sociedad y se puede ir logrando aunque sea un poco la confianza hacia las instituciones públicas.

En el mismo contexto, es interesante lo planteado por Vergara (2008:28) "En los últimos años nos encontramos con que diversos organismos internacionales como la OCDE y el Banco Mundial han comenzado a presionar a los gobiernos nacionales para transparentar su información y explicar sus decisiones". Esto por considerar que muchas de las crisis y decisiones gubernamentales equivocadas, se hubieran podido evitar si la sociedad tuviera el conocimiento de la información pública.

La transparencia como cultura política y ciudadana, encuentra sus principales razones en el sistema político mexicano. Esto en virtud, de que enfrenta una crisis de credibilidad y de representatividad, lo cual se originó debido principalmente a las experiencias de corrupción y 
clientelismo político que ha vivido el país a lo largo de su historia.

De tal forma, que al no tener los ciudadanos confianza en las instituciones públicas, se refleja en el desinterés de inversión privada para reactivar la economía nacional y en la evasión de impuestos aduciendo que no se utilizan para el bien común, entre otros. Por ello, la transparencia se presenta como estrategia para construir la confianza de los ciudadanos.

Se considera, que solamente con la participación de la sociedad, se puede contribuir a la formación de los servidores públicos que deben estar comprometidos a respetar las leyes y con la cultura de honestidad y fortalecimiento de una ética pública a favor de los ciudadanos.

En ese tenor, es importante compartir lo planteado Arenilla Sáez (2014:92)

La finalidad es lograr ciudadanos que entiendan mejor los asuntos públicos y sean más exigentes con sus representantes y dirigentes políticos y dirigentes políticos más responsables. En definitiva, el ejercicio de la transparencia supone la redistribución del poder actual de los dirigentes políticos entre los ciudadanos y un mayor empoderamiento de estos.

En esa tesitura, considerando lo señalado por Arenilla, tanto la activa participación de los ciudadanos, como la transparencia y el derecho de acceso a la información, representa un avance significativo ene todos los países del mundo.

En México, con la entrada en vigor de la Ley de Transparencia, se obliga a las instituciones del gobierno a proporcionar a los ciudadanos la información que les interese sobre la gestión gubernamental, el uso de los recursos públicos que puede incluir el presupuesto asignado a las Instituciones que forman parte del gobierno federal, estatal y municipal y los informes de su ejecución, entre otra información financiera.

Al concientizar a los ciudadanos sobre lo importante de su participación y al hacer efectivo el derecho de acceso a la información, se podrá influenciar para que la toma de decisiones, así como, la formulación, instrumentación, monitoreo y evaluación de políticas públicas sobre la acción gubernamental se lleve a cabo apegadas a las leyes.

Aunado a ellos Merino (2018:18) comparte lo siguiente:

El conocimiento de los asuntos públicos que favorece la transparencia ha hecho posible que, cada vez más, los ciudadanos organizaciones sociales, empresariales, académicas y medios de comunicación monitoreen las acciones y decisiones de los gobiernos y esa observación permanente desincentiva los intercambios corruptos.

Merino, señala que la participación activa de los ciudadanos y las organizaciones civiles coadyuvan a que haya menos comportamientos corruptos.

Sin duda, en México están emergiendo nuevas formas de participación ciudadana, los mexicanos exigen un buen gobiernos.

En el ejercicio ciudadano del derecho de acceso a la información, se han constituido organizaciones civiles que promueben la transparencia, el derecho de acceso a la información y la rendición de cuentas. En ese sentido a finales del año 2002, se formó una S.C. 
que se denomina Colectivo por la Transparencia donde se integraron 6 organizaciones de la sociedad civil. En 2018 estaba integrada por 16 organizaciones de la sociedad civil. En abril de 2021 se buscó la integración y no se encontró en páginas electrónicas información sobre su integración.

Dando importancia a la participación de la sociedad civil, en los últimos años en México se promueve la creación de organizaciones civiles y para ello se ha creado normatividad que entre otras cosas permite identificar y conocer los objetivos que tiene cada una de ellas. Así mismo se creó en la Ley Federal de Fomento a las Actividades de las Organizciones de la Sociedad Civil, el Registro Nacional de instituciones y organizaciones políticas sociales y civiles.

En ese tenor, es necesario que se promueba la creación de organizaciones de participación ciudadana como mecanismos fundamentales para la participación en el diseño de las políticas públicas.

Con ello, se busca impulsar a los ciudadanos para que participen de forma activa en el monitoreo, el seguimiento y la evaluación de la gestión pública, para establecer medidas correctivas dentro de la función pública, ya sea para proponer mejoras en la administración o en la sanción ante actos de corrupción.

Para implementar la estrategia se requiere construir alianzas con la sociedad civil y el establecimiento de foros permanentes de consulta, evaluación y seguimiento sobre la gestión gubernamental.

En ese tenor, Egaña Baraona (2015:67) señala lo siguiente:
Esta mirada desde la complejidad del entorno obliga también a comprender los actores que juegan un rol relevante en dichas instituciones, los grupos sociales o de ciudadanos con quienes deberán interactuar, los sistemas de participación que deberán tener, las normas éticas y de probidad a las cuales estarán sometidas, la transparencia a la cual estarán obligadas, el sistema político que tendrá influencia en cada institución, junto con el régimen de vinculación con autoridades superiores de gobierno, tanto a niveles centrales, regionales o locales.

Como se puede apreciar el entorno es complejo y solo interactuando los servidores públicos en representación de las instituciones y los grupos de ciudadanos, se avanzarán a un mejor desempeño del gobierno.

También, Villoria (2016:23), señala lo siguiente: Cuando la transparencia es real y funciona como valor de referencia, es un antídoto claro contra la corrupción, sobre todo porque permite detectar mejor los casos existentes y, con ello, desincentiva dichas actividades, más aún si la persecución y sanción siguen a la detección previa. Hoy en día, a través de las normas de acceso a la información, la publicidad activa, las apps y los wikis en el marco del denominado Gobierno abierto, los efectos positivos de la transparencia han aumentado.

Interesante la aportación de Villoria, en el sentido que establece el resultado de una transparencia real que abona a la disminución de conductas corruptas, también señala que las 
aplicaciones digitales que nos ofrecen dentro del gobierno abierto condicionan efectos positivos en los ciudadanos.

Por otro lado, en los últimos años los procesos gubernamentales al igual que las actividades en la iniciativa privada, se ha multiplicado la interacción internacional y eso da lugar a la necesidad de considerar los requisitos que se establecen para realizar gestiones, en diferentes organismos internacionales como la Organización para la Cooperación y el Desarrollo Económicos y el Banco Mundial, México tiene enormes retos que lograr en especial la rendición de cuentas, siendo el derecho de acceso a la información un pilar fundamental.

En este sentido, Vergara (2008:33) señala que:

Todo esto, requiere el diseño de estrategias que tengan como objetivo dar cumplimiento a tan importante derecho y como el Estado mexicano otorga la garantía y está plasmado en la Constitución, no debe evadirse ni posponer.

Por ello, es necesario realizar el diseño de estrategias para cumplir con las obligaciones de transparencia y acceso a la información y con ello mejorar la confianza hacia el gobierno.

\section{METODOLOGÍA}

Al realizar una investigación, es primordial establecer la metodología, ya que permitirá elaborar, definir y sistematizar las técnicas, métodos y procedimientos que se deben seguir durante el desarrollo de un proceso de investigación para la producción de conocimiento.

En este sentido, por el tema a investigar, se eligió el enfoque cualitativo y su utilizó el método descriptivo, porque permite detallar la estructura legal del derecho de acceso a la información, el contexto legal de la participación ciudadana y se investiga las solicitudes que se reciben en el Instituto Nacional de Transparencia y Acceso a la Información Pública y Protección de Datos Personales (INAI) y los 10 sujetos obligados que recibieron mas solicitudes desde 12 de junio de 2003 hasta Septiembre de 2020.

Dentro de los procedimientos de esta investigación se revisaron diversas referencias bibliográficas como leyes, libros, artículos en revistas especializadas, páginas electrónicas de organismos nacionales e internacionales, instituciones gubernamentales y de organizaciones civiles.

Con todo ello, se estructuraron los hallazgos de forma ordenada, se formularon los objetivos y las preguntas de investigación. Así mismo, se integró la información de especialistas en el marco teórico y por último se presentan los resultados y las conclusiones.

\subsection{Objetivos de investigación}

1. Analizar los antecedentes y evolución del derecho de acceso a la información pública.

2. Revisar la estructura jurídica del derecho de acceso a la información pública

3. Recopilar el total de solicitudes recibidas y los 10 sujetos obligados que recibieron 
el mayor número de solicitudes de información pública.

\subsection{Preguntas de investigación}

1. ¿Cuáles son los antecedentes y evolución del derecho de acceso a la información pública?

2. ¿Cuál es la estructura jurídica del derecho de acceso a la información pública?

3. ¿Cuántas solicitudes se recibieron y los 10 sujetos obligados que recibieron el mayor número de solicitudes de información pública?

\section{RESULTADOS}

Después de analizar la temática que nos ocupa, se llegaron a los siguientes resultados.

Considerando la problemática, nos permite identificar que una de las estrategias debe ser el fomento a los valores en los servidores públicos, en virtud de que al ejercer el derecho de acceso a la información, se sientan vigilados.

Así también, al cumplir con la obligación de proporcionar información cuando le sea solicitada, le ofrece un beneficio porque podrá limitar la participación en hechos que pudieran poner en riesgo su trabajo como servidor público y además puede hacerse acreedor a sanciones por no cumplir con las disposiciones de las leyes.

Por la forma de interactuar entre los ciudadanos y el gobierno a través de plataformas digitales, es importante también señalar lo que comparte INEGI, según los datos de la ENDUTIH 2019, el 56.4 por ciento de los hogares del país disponen de conexión a internet por lo que tenemos que el $43.6 \%$ de los hogares no cuentan con internet y por tanto no pueden interactuar con el gobierno y solicitarle información.

Otro de los hallazgos, es que en México los ciudadanos enfrentan una crisis de credibilidad y de representatividad, lo cual se originó debido principalmente a las experiencias de corrupción y clientelismo político que ha vivido el país a lo largo de su historia.

Por lo tanto, a medida que el gobierno publique la información de forma clara y oportuna se pondrá un freno a la comisión de actos deshonestos o decisiones discrecionales de parte de los servidores públicos.

El gobierno mexicano enfrenta muchos retos, quizá de mayor prioridad que el brindar acceso a la información pública, dentro de esos retos se pueden señalar los siguientes: el aumento progresivo de las personas que viven en pobreza, la inseguridad en todo su territorio, los procesos electorales constosos, los escandalos de corrupción, entre otros.

Sin embargo, la transparencia y acceso a la información permea en cada uno de ellos, ya que si hay información real que no haya sido manipulada con intereses políticos se conocerá a ciencia cierta el número de mexicanos que viven en pobreza, que apoyos gubernamentales reciben y que requisitos solicita el gobierno para entregar dichos apoyos.

Las modificaciones a la Constitución Política de los Estados Unidos Mexicanos en materia de transparencia, han contribuido a tener un panorama con políticas de apertura desde dos angulos; el primero; la política que obliga a proporcionar la información necesaria para conocer el desempeño del gobierno y la segunda; 
para promover la participación de la sociedad civil.

Al revisar el ejercicio del derecho de acceso a la información, se encontró el número de solicitudes que ha recibido el órgano garante desde junio de 2003 hasta septiembre de 2020 y se puede identificar que año con año se incrementan las solicitudes de información pública. En el siguiente cuadro se puede conocer el número de solicitudes de información del ámbito el federal.

Cuadro 1: Solicitudes de información pública de 2003 a 2020.

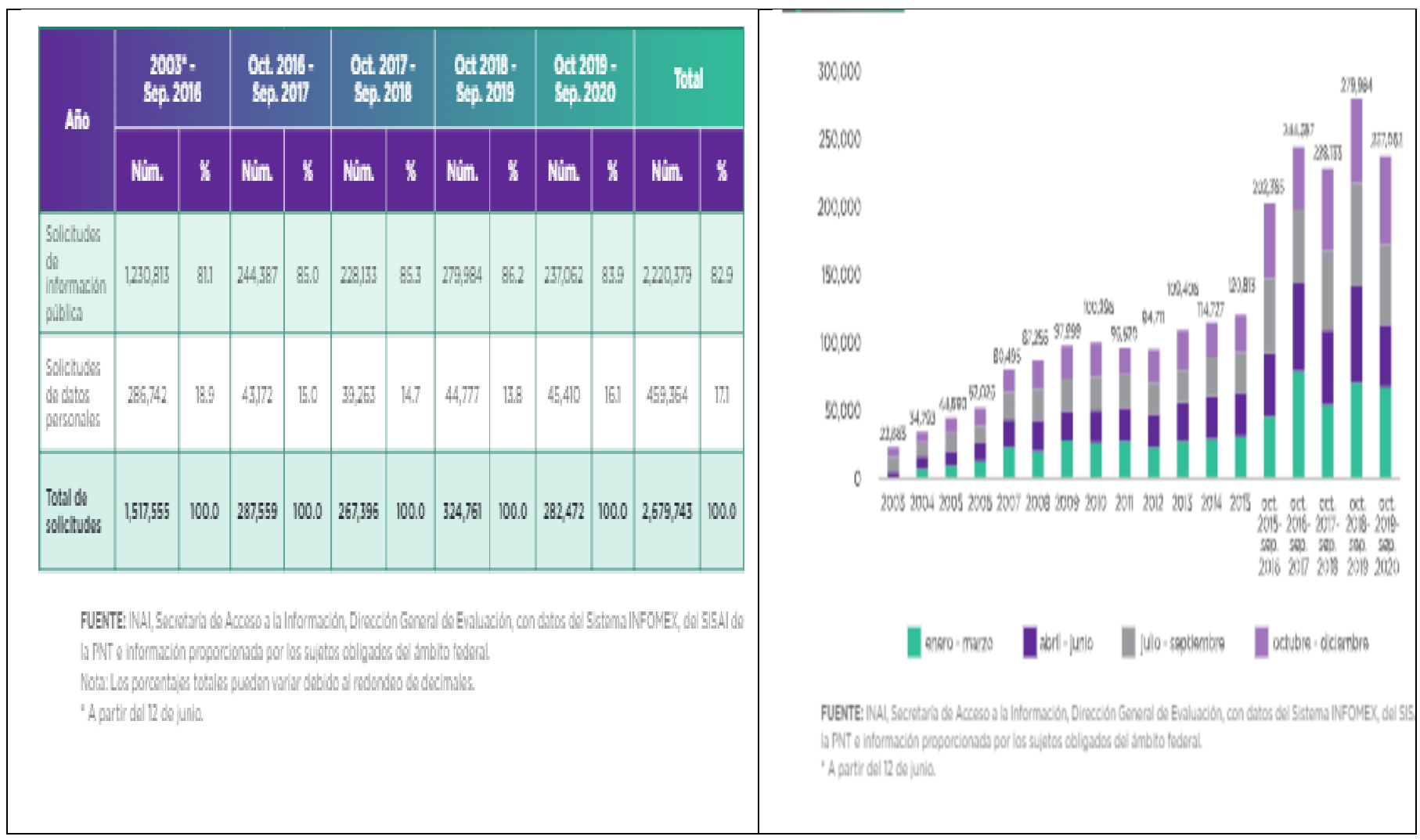

Fuente: Información recuperada del Informe del INAI 2020 página 22 localizado en:

https://micrositios.inai.org.mx/informesinai/?page_id=519

En el cuadro y gráfica anterior, se puede observar que año tras año se van en incremento las solicitudes de información pública que realizan los ciudadanos a las instituciones gubernamentales. El incremento mayor se presentó de octubre de 2018 a septiembre de 2019, donde se recibieron 324,761 posteriormente disminuyen las solicitudes de octubre de 2019 a septiembre de 2020 con un total de 282,472 .

Es importante también señalar que desde 2003 hasta 2020 se han recibido un total de 2’679,743, solicitudes de información.

A continuación se presenta el cuadro y la grafica de los 10 sujetos obligados a nivel federal 
que recibieron el mayo número de solicitudes de

información.

Cuadro 2: Solicitudes por Sujetos Obligados

\begin{tabular}{|r|l|r|}
\hline & \multicolumn{1}{|c|}{$\begin{array}{c}\text { Solicitudes recibidas por sujeto obligado } \\
\text { de 2003 a 2020 }\end{array}$} & \\
\hline & \multicolumn{1}{|c|}{$\begin{array}{c}\text { Sujeto obligado } \\
\text { recibidas }\end{array}$} \\
\hline 1 & Suprema Corte de Justicia de la Nación & 203,792 \\
\hline 2 & Instituto Mexicano del Seguro Social & 136,122 \\
\hline 3 & Secretaría de Educación Pública & 81,596 \\
\hline 4 & $\begin{array}{l}\text { Comisión Federal para la Protección } \\
\text { Contra Riesgos Sanitarios }\end{array}$ & 66,688 \\
\hline 5 & Secretaria de Salud & 64,132 \\
\hline 6 & $\begin{array}{l}\text { Secretaría de Medio Ambiente y Recursos } \\
\text { Naturales }\end{array}$ & 58,096 \\
\hline 7 & Secretaria de Hacienda y Crédito Público & 54,228 \\
\hline 8 & $\begin{array}{l}\text { Instituto de Seguridad y Servicios Sociales } \\
\text { de los Trabajadores del Estado }\end{array}$ & 50,245 \\
\hline 9 & Secretaría de Comunicaciones y Transporte & 49,157 \\
\hline 1 & \multicolumn{2}{|l}{} \\
0 & Secretaría de la Función Pública & 46,803 \\
\hline
\end{tabular}

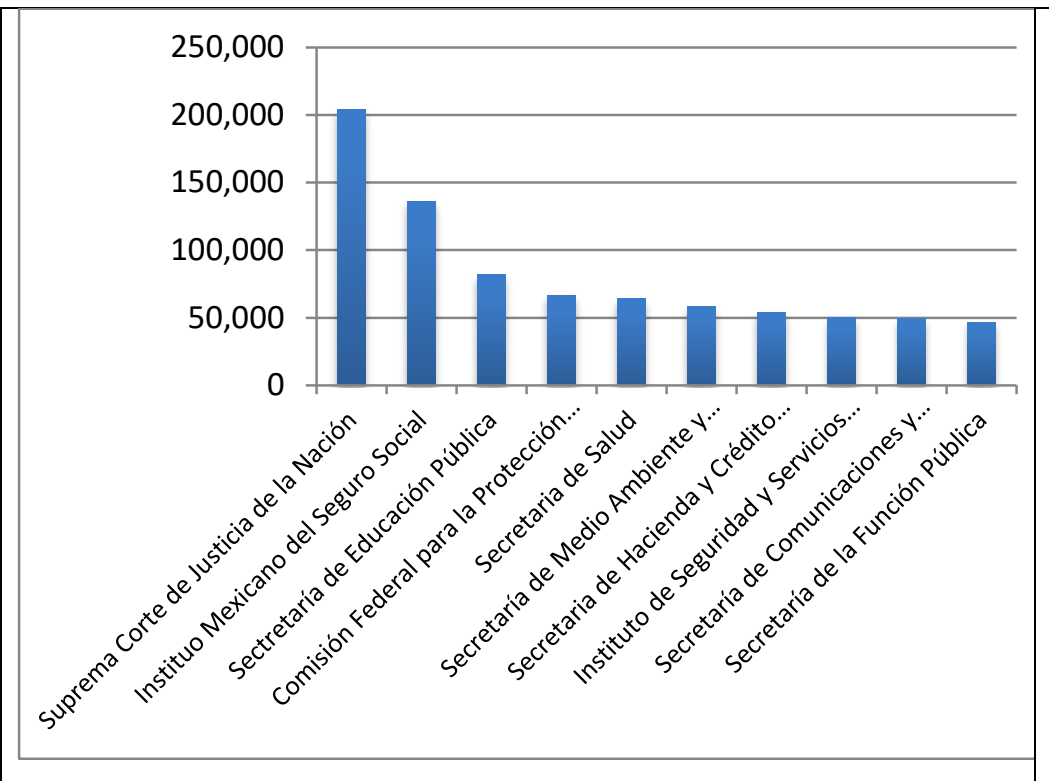

Fuente: Elaboración propia con datos del informe del INAI 2020. página 27 localizado en:

https://micrositios.inai.org.mx/informesinai/?page_id=519

Resulta interesante conocer que la Suprema Corte de Justicia de la Nación recibe una mayor cantidad de solicitudes y en segundo lugar el Instituto Mexicano del Seguro Social.

\section{Conclusiones}

Con la entrada en vigor de la Ley de Transparencia, las organizaciones del gobierno federal y las entidades federativas, tienen la obligación de dar a conocer la información sobre su desempeño con ello, México se encamina hacia un gobierno abierto, flexible y transparente, que promueve la cultura ciudadana.
Se transita por nuevos caminos y aunque esos caminos son resultado de exigencias internacionales, sin duda las modificaciones a las leyes de transparencia donde se describe de manera detallada las obligaciones de transparencia y acceso a la información, proporciona certidumbre jurídica a los ciudadanos. De cumplirse a cabalidad dichas obligaciones, se propicia que los ciudadanos recuperen la confianza en el gobierno.

Las modificaciones a la Constitución Política de los Estados Unidos Mexicanos en materia de transparencia, han contribuido a tener un panorama con políticas de apertura desde dos 
angulos; el primero; la política que obliga a proporcionar la información necesaria para conocer el desempeño del gobierno y la segunda; para promover la participación de la sociedad civil.

La sociedad mexicana, ha respondido de manera lenta, todavía no se tiene claro los beneficios de ejercer el derecho de acceso a la información. Sin embargo a medida que se resalte la importancia de la rendición de cuentas y el promover las conductas de los servidores públicos que se comprometan a la construcción de una mejor sociedad.

Con el cumplimiento de las obligaciones establecidas en la Ley de transparencia y el acceso a la información, se pondrá un freno a la comisión de actos deshonestos o decisiones discrecionales de parte de los servidores públicos, para con ello contribuir a la credibilidad y propicien que las instituciones gubernamentales cumplan sus objetivos y no se permita privilegiar los intereses privados sobre el bien común de la sociedad.

Para construir un mejor país, es necesario ser conscientes de que cada mexicano se debe convertir en agente de cambio y entre todos difundir el derecho que tienen los ciudadanos a la información pública gubernamental de manera oportuna, suficiente y garantizada, así como la participación de la sociedad en el análisis y evaluación en el desempeño del gobierno.

En relación al marco legal de la transparencia y el derecho de acceso a la información se considera inacabado, por el hecho de requerir constantes ajustes para dar respuesta a un tejido social cambiante. Sin embargo, se identificó que todavía se deberán realizar modificaciones a leyes secundarios que fortalezcan y brinden certidumbre jurídica a los ciudadanos. Como ejemplo de esas leyes se pueden mencionar la Ley Federal de Archivos, la Ley General de Contabilidad Gubernamental, la Ley de Fiscalización y Rendición de Cuentas, la Ley Federal de Presupuesto y Responsabilidad Hacendaria, entre otras.

Sin embargo, lo más importante para obtener resultados satisfactorios en la transparencia, derecho de acceso a la información pública es la voluntad política de los servidores públicos y que tanto el poder ejecutivo como el legislativo realicen acciones que coadyuven a mejorar en el cumplimiento de las obligaciones de transparencia y de acceso a la información.

Aunque, se sabe que no son suficiente las leyes, se debe avanzar en el diseño e implementación de estrategias encaminadas a vencer las resistencias burocráticas que han desafiado a toda la estructura jurídica.

Es importante recordar que se requieren debates abiertos y libres con la participación de integrantes del gobierno y de la sociedad civil. En especial en México donde la desigualdad es uno de los principales problemas que enfrenta la población. Por ello, las organizaciones de la sociedad civil tienen un grn compromiso ya que pueden promover y buscar satisfacer las necesidades de la población vulnerable, donde fomenten el impulso de una ciudadanía plena y responsable. 


\section{REFERENCIAS}

Arenilla Saez, M. (2014) Transparencia, Accesibilidad y Rendición de Cuentas. En Administración 2032. Teclas para transformar la Administración Pública Española. Grupo de Investigación en Administración Pública 2032. GIAP 2032. . España. INAP

Constitución Política de los Estados Unidos Mexicanos,

Egaña Baraona, R. (2015) Fortalecimiento Institucional: Una mirada desde la experiencia. En 20 años de Congresos CLAD. Aportes a la Reforma del Estado y la Administración Pública en Iberoamérica. Venezuela. CLAD.

Ley Federal de Transparencia y Acceso a la Información Pública Gubernamental. Recuperada en: www.diputados.gob.mx/LeyesBiblio/pdf/1_100715.pdf

Ley Federal de Fomento a las actividadaes de las organizaciones de la Sociedad Civil

Ley General de Transparencia y Acceso a la Información Pública. Localizada en: www.diputados.gob.mx/LeyesBiblio/pdf/244_181215.pdf

Luna, P. I. (2009). Movimiento social del derecho de acceso a la información en México. México. Instituto de investigaciones jurídicas (IIJ). UNAM

Merino, M. (2008) La transparencia como política pública. En Ackerman (Coord). Más allá del acceso a la información. Transparencia, rendición de cuentas y Estrado de Derecho. México. Siglo XXI

Quintanilla Gabriela y Gil García José Ramón. (2016) Gobierno abierto y datos vinculados: conceptos, experiencias y lecciones con base en el caso de mexicano. En la Revista Reforma y Democracia. $\mathrm{N}^{\circ}$. 65. Venezuela. CLAD.

Vergara, R. (2008). La Transparencia como problema. Serie de Cuadernos de transparencia 05. México 5ta. ed. IFAI.

Villoria Mendieta M. (2016) El papel de la Administración Pública en la generación de calidad democrática. En la Revista Reforma y Democracia No. 65, Jun. 2016. Venezuela. CLAD.

\section{Páginas consultadas de internet}

www.diputados.gob.mx/LeyesBiblio/pdf/1_100715.pdf

https://micrositios.inai.org.mx/informesinai/?page_id=519

https://www.gob.mx/indesol/acciones-y-programas/avisosc-avisos-de-las-organizaciones-de-la-sociedad-civil https://www.inegi.org.mx/contenidos/saladeprensa/boletines/2021/EstSociodemo/ResultCenso2020_Nal.pdf https://www.inegi.org.mx/contenidos/saladeprensa/aproposito/2020/eap_internet20.pdf

https://www.gob.mx/indesol/acciones-y-programas/registro-federal-de-las-osc 